\title{
Draft genome sequence of the strain 16-537536, isolated from a patient with bronchiectasis and its relationship to the Pseudomonas koreensis group of the Pseudomonas fluorescens complex
}

\author{
Ad. C. Fluit ${ }^{1^{*}} \mathbb{0}$, Malbert R. C. Rogers ${ }^{1}$, María Díez-Aguilar ${ }^{2,3}$, Rafael Cantón², Barry J. Benaissa-Trouw', \\ Jumamurat R. Bayjanov ${ }^{1}$ and Miquel B. Ekkelenkamp ${ }^{1}$
}

\begin{abstract}
Objective: The Pseudomonas koreensis group bacteria are usually found in soil and are associated with plants. Currently they are poorly described. Here we report on the whole genome sequence of a bacterial isolate from a patient with bronchiectasis that was first identified as P. koreensis, and on its position in the P. koreensis group.

Results: Strain 16-537536 was isolated from a patient with bronchiectasis from Spain and initially identified by MALDI-TOF as P. koreensis, a member of the Pseudomonas fluorescens complex. However, the average nucleotide identity analysis (ANIb) and whole genome alignments of the draft genome sequence of this strain showed it to be a member of the $P$. koreensis group of the $P$. fluorescens complex, but belonging to an undescribed species. In addition, based on ANIb analysis, the P. koreensis group contains several other unnamed species. Several genes for putative virulence factors were identified. The only antibiotic resistance gene present in strain 16-537536 was a class $C$ $\beta$-lactamase. The correct identification of bacterial species from patients is of utmost importance in order to understand their pathogenesis and to track the potential spread of pathogens between patients. Whole genome sequence data should be included for the description of new species.
\end{abstract}

Keywords: Pseudomonas fluorescens, Bronchiectasis, Whole genome sequencing, Taxonomy, Pseudomonas koreensis

\section{Introduction}

The Pseudomonas koreensis group is classified within the Pseudomonas fluorescens complex and consist of several species, including Pseudomonas koreensis [1, 2]. P. fluorescens complex isolates have been recovered from many environmental sources, and are frequently associated with plants and soils [3]. P. koreensis was first isolated

\footnotetext{
*Correspondence: a.c.fluit@umcutrecht.nl

${ }^{1}$ Department of Medical Microbiology, University Medical Center Utrecht,

Room G04.614, PO Box 85500, 3508 GA Utrecht, The Netherlands

Full list of author information is available at the end of the article
}

from Korean agricultural soils. Subsequently, it has been isolated from rhizospheres, other agricultural soils $[4,5]$, and from yak milk [6]. It has also been found as a pathogen in freshwater fish [7]. The bacterium has been reported to produce antimicrobial and antifungal compounds [8-10]. Here we report the sequence of $P$. koreensis group strain 16-537536 from a bronchiectasis patient and on its relationship to other strains of this group for which whole genome sequence data are available.

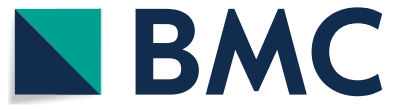

(c) The Author(s) 2019. This article is licensed under a Creative Commons Attribution 4.0 International License, which permits use, sharing, adaptation, distribution and reproduction in any medium or format, as long as you give appropriate credit to the original author(s) and the source, provide a link to the Creative Commons licence, and indicate if changes were made. The images or other third party material in this article are included in the article's Creative Commons licence, unless indicated otherwise in a credit line to the material. If material is not included in the article's Creative Commons licence and your intended use is not permitted by statutory regulation or exceeds the permitted use, you will need to obtain permission directly from the copyright holder. To view a copy of this licence, visit http://creativecommons.org/licenses/by/4.0/. The Creative Commons Public Domain Dedication waiver (http://creativecommons.org/publicdomain/zero/1.0/) applies to the data made available in this article, unless otherwise stated in a credit line to the data. 


\section{Main text Methods}

Strain 16-537536 was a respiratory isolate from a Spanish patient (50-70 years old) with Karthagener syndrome and bi-basal bronchiectasis in October 2015. It was cultured from a sputum sample by inoculation on $5 \%$ sheep blood, chocolate and MacConkey agar plates. Leukocytes and Gram-negative rods were observed in the Gram-stain of the sputum sample. The agar plates were incubated at $37{ }^{\circ} \mathrm{C}$ in aerobic and $5 \% \mathrm{CO}_{2}$ atmosphere conditions; and significant bacterial growth was detected on all the plates after $24 \mathrm{~h}$. Identification and susceptibility testing was performed with the MicroScan Walkaway system (Beckman Coulter), which identified the isolate as Pseudomonas spp. The isolate was also analyzed by MALDI-TOF (Bruker, Germany). Briefly, the isolate was spotted in duplo on a steel target and a $\alpha$-cyano-4-hydroxy-cinnamic acid matrix was applied. The spectra which were obtained were compared to those in the Bruker library. Bacterial DNA was isolated with the QIAcube DNeasy Blood and Tissue Kit using the bacterial or yeast DNA with enzymatic lysis protocol (Qiagen, Germany) after pretreatment with $3 \mu \mathrm{g} / \mathrm{ml}$ lysozyme for $30 \mathrm{~min}$ at $37{ }^{\circ} \mathrm{C}$. A DNA library was prepared using the Illumina Nextera XT kit with the corresponding protocol (Illumina, CA) and subsequently sequenced on an Illumina NextSeq platform using the $2 \times 150$ bp sequencing kit.

Contigs were assembled with SPAdes genome assembler v.3.6.2. Contigs longer than 500 bp with at least tenfold coverage were analyzed further.

The relationship to closely related strains was estimated by establishing an average nucleotide identity score based on BLAST (ANIb) [11, 12], using the whole genome sequences of the $P$. koreensis group strains as previously defined by Garrido-Sanz et al. [13]. In addition, we included the type strain (LMG 21318), two other strains identified as P. koreensis (CRS05-R5 and D26) (NCBI GCF_900101414.1, CP015852 and CP014947.1, respectively), and Pseudomonas baetica (NCBI PKLC01000000) [14].

The assembled contigs were separately annotated using RAST [15] and Prokka [16]. Further analysis for the presence of resistance genes was performed with ResFinder 3.1 of the Center for Genomic Epidemiology (DTU, Denmark) [17].

\section{Results}

The assembly resulted in 348 contigs with a total length of $6,613,537 \mathrm{bp}$. The average coverage was 52 -fold and the GC-content was $59.9 \%$.
Primary identification was performed by MALDITOF. The top 5 results with their score for the first spot were: P. koreensis (1.99); P. koreensis (1.89); Pseudomonas jessenii (1.85); Pseudomonas azotoformans (1.82); Pseudomonas vancouverensis (1.8) and for the second spot: P. koreensis (2.03) P. jessenii (1.89); Pseudomonas corrugate (1.88); P. koreensis (1.84); P. vancouverensis (1.82). The single score above 2.00 led to a presumptive identification of the isolate as $P$. koreensis. However, whole genome alignments suggested that $P$. koreensis 16-537536 might belong to a different species. To verify the species identification, an ANIb was performed, using the whole genome sequences of the $P$. koreensis group strains defined by Garrido-Sanz et al. [13]. In addition we included the type strain (LMG 21318) and the sequence data of four other strains identified as $P$. koreensis and available at NCBI (CRS05-R5, $\mathrm{P} 2$, Ab36, and D26). When two isolates have an ANIb identity score below $95-96 \%$, they are generally considered to be separate species [11]. Applying a conservative cut-off of $95 \%$, the $P$. koreensis group can be divided into 21 different species (Fig. 1). The ANIb confirms that strain 16-537536 and the P. koreensis type strain belong to different, albeit very closely related species. Strain 16-537536 clusters with two strains labeled $P$. fluorescens AU5633 and Pseudomonas spp. W15FEB9B. The strain identified as $P$. koreensis CRS05-R5 clusters with the type strain, whereas D26 strain clusters with four other strains, including one that was identified as Pseudomonas moraviensis. Strain Ab36 clusters with $P$. fluorescens SF39a. Strain P2 is unrelated to any of the strains tested in the ANIb, with the lowest score when compared to any of the other strains $(<80.3 \%)$.

Gene annotation using RAST identified 6194 protein coding sequences and 78 sequences encoding RNAs. An intrinsic class $C \beta$-lactamase was identified, but neither RAST nor ResFinder yielded any additional (acquired) antibiotic resistance genes. Based on RAST annotation, there was no evidence for plasmids or transposons. However, alignment against plasmid 4 of $P$. koreensis strain P19E3 (GenBank Acc. No CP027481) showed that $69 \mathrm{~kb}$ of DNA encoding putative heavy metal/copper resistance related sequences were also present on the approximately $283 \mathrm{~kb}$ P19E3 plasmid. Virulence factors could not be identified using RAST. Annotation with Prokka yielded similar results. Further analysis using BLAST against GenBank identified several putative virulence factors. Although $P$. koreensis group strains are usually not pathogenic in humans, they have been reported as pathogens in freshwater fish [6]. Based on RAST, several potential virulence factors were encoded: enzymes involved in alginate biosynthesis, type II and type VI secretion systems, toxins, pyocins, two filamentous hemagglutinins, 


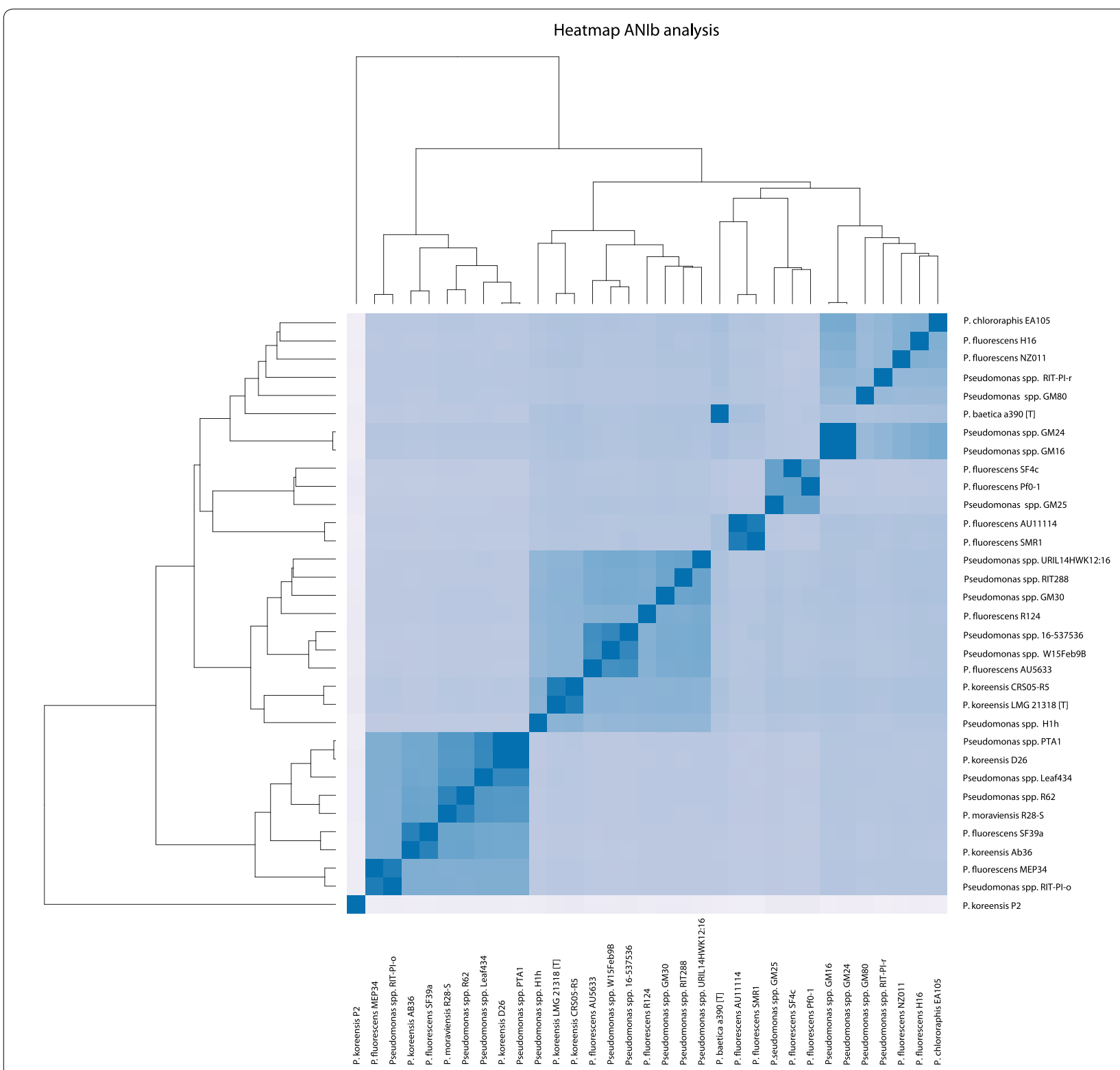

Fig. 1 Heatmap of the ANIb of isolates belonging to the P. koreensis group of the P. fluorescens complex. The identity increases form 85 to $86 \%$ (light lavender) to 100\% (dark blue). Type strains are indicated by [T] behind the species name. For details of the percentages see Additional file 1: Figure S1

urease, fimbriae, a thermostable hemolysin, a flagellum, a microcin, and a bacteriocin.

\section{Discussion}

Strain 16-537536 has a comparable genome size to other P. koreensis strains: 6,622,028 bp for CI12, 5,991,224 bp for CRS05-R5, and 6,444,290 bp for the P19E3 chromosome and 1,053,904 bp for its four plasmids ([4, 18]; GenBank Acc. No. CP027477-CP027481).

The results from the ANIb analysis are comparable to those described by Garrido-Sanz et al. [13], with the exception of the strains Pseudomonas spp. RIT-PI-o and P. fluorescens MEP34, which clustered separately in the study of Garrido-Sanz et al. [13]. The reason for this discrepancy is not clear, but differences between isolate sets may play a role. The ANIb confirms that strain 16-537536 and the $P$. koreensis type strain belong to different, albeit very closely related species.

Our difficulties with identifying strain 16-537536 to the species level, despite having its whole genome sequence available, illustrates the needs for improvement of the the P. koreensis group taxonomy. In addition, we found three 
more strains, currently identified as $P$. koreensis, which potentially represent several novel species. Although the majority of the strains used in our analysis are plantassociated, two isolates were obtained from cystic fibrosis patients and our strain was retrieved from a patient with bronchiectasis; correct species assignment will aid in understanding the epidemiology and pathogenesis of Pseudomonas spp. in these diseases. The availability of whole genome sequences of the type strains of all species within the $P$. fluorescens complex would greatly contribute to this objective, as it would allow correct assignment of already sequenced strains and would support the designation of novel species.

The presence of hemolysin has been reported previously for Pseudomonas aeruginosa and its activity is considered an important virulence factor in infection [19].

Filamentous hemagglutinin has been shown to be an iron-reponsive virulence factor in the $P$. fluorescens strain TSS, which is pathogenic for fish [20]. A mutant of $P$. fluorescens TSS, in which filamentous hemagglutinin was inactivated, showed less biofilm production and extracellular matrix, had no apparent flagella and motility, was defective in attachment to host cells, showed no selfaggregation, exhibited less ability for hemagglutination and had a reduced survival in serum. In vivo experiments in fish showed attenuation of dissemination in tissue by the mutant strain, and reduced host mortality.

Urease has been implicated as a virulence factor during human respiratory tract infection by Haemophilus influenzae [21]; the enzyme enhances viability in an acid environment. The urease of strain 16-537536 may play a similar role, especially considering that this strain was isolated from the sputum of a bronchiectasis patient with a lung infection. Based on similarity with proteins in Pseudomonas species, strain 16-537536 encodes four potential toxins, one of which was annotated previously as insecticidal toxin complex TcaB2. Additional information for these toxins is lacking and their roles in virulence remains unknown [GenBank accession numbers WP_108183637.1, WP_108183629.1, WP_108183629.1, WP_108181890.1, and WP_108183711.1].

The presence of type II and type VI secretion systems in strain 16-537536 may indicate that the bacterium expresses virulence factors which are excreted by these systems. The type II secretion system of $P$. aeruginosa is a general secretion pathway that secretes virulence factors, such as guanylate cyclase ExoA and the proteases LasA/B; it is also present in other pathogenic bacteria [22, 23]. The type VI secretion systems are used by many Gramnegative bacteria to inject toxic effector molecules into eukaryotic or prokaryotic cells and have a role in eliminating other bacteria that occupy the same niche. This has for example been shown for Salmonella typhimurium in an animal model [24]. Pyocins, microcins, and bacteriocins, which are antibacterial peptides, may also contribute to this process [25]. Finally, fimbriae and flagella may contribute to virulence by facilitating adhesion and providing mobility.

Besides these putative virulence factors, additional virulence factors in strain 16-537536 may not been have been identified because annotation of the genome is not complete; many open reading frames were identified as hypothetical proteins. Furthermore, it is unknown whether the identified virulence factors are indeed expressed during human colonization and infection.

\section{Conclusion}

Strain 16-537536 is a member of the P. koreensis group and belongs to a novel, currently undescribed species. The strain encodes several putative virulence factors. It has an intrinsic AmpC $\beta$-lactamase, but no additional acquired antibiotic resistance genes.

\section{Limitations}

- WGS of type strain not always available.

- Annotation of bacterial genes incomplete.

\section{Supplementary information}

Supplementary information accompanies this paper at https://doi. org/10.1186/s13104-019-4863-2.

Additional file 1: Figure S1. Heatmap of the ANIb of isolates belonging to the $P$. koreensis group of the $P$. fluorescens complex with percentage identity. Type strains are indicated by $[T]$ behind the species name.

Abbreviation

ANIb: average nucleotide identity analysis.

Acknowledgements

Not applicable.

Authors' contributions

AF design, analysis and writing; MR and JB analysis and writing; MD, RC, and ME study design; BB experiments and analysis. The authors read and approved the final manuscript.

\section{Funding}

The research leading to these results has received support from the Innovative Medicines Initiative Joint Undertaking under Grant Agreement No. [115721-2], resources of which are composed of financial contribution from the European Union's Seventh Framework Programme (FP7/2007-2013) and EFPIA companies in kind contribution. MD-A was also partially supported by the Fundación Francisco Soria Melguizo (Madrid, Spain). The sponsors or funders were not involved in the study or the preparation of the manuscript.

\section{Availability of data and materials}

This whole-genome shotgun project has been deposited at ENA under the project number PRJEB30245. The version described in this paper is the first version. 


\section{Ethics approval and consent to participate}

Spanish law and ethics rules do not require patient consent for the use of recovered strains or for the use of patient data when this is anonymised and cannot be traced back to the individual patient. The Medical Ethical Committee of Hospital Universitario Ramón y Cajal approved the study.

\section{Consent for publication}

Not applicable.

\section{Competing interests}

The authors declare that they have no competing interests.

\section{Author details}

${ }^{1}$ Department of Medical Microbiology, University Medical Center Utrecht, Room G04.614, PO Box 85500, 3508 GA Utrecht, The Netherlands. ${ }^{2}$ Servicio de Microbiología, Hospital Universitario Ramón y Cajal, Instituto Ramón y Cajal de Investigación Sanitaria (IRYCIS), Madrid, Spain. ${ }^{3}$ Red Española de Investigación en Patología Infecciosa (REIPI), Madrid, Spain.

Received: 18 October 2019 Accepted: 16 December 2019

Published online: 06 January 2020

\section{References}

1. Gomila M, Peña A, Mulet M, Lalucat J, García-Valdés E. Phylogenomics and systematics in Pseudomonas. Front Microbiol. 2015;6:214. https://doi. org/10.3389/fmicb.2015.00214.

2. Garrido-Sanz D, Meier-Kolthoff JP, Göker M, Martín M, Rivilla R, RedondoNieto M. Genomic and genetic diversity within the Pseudomonas koreensis group. PLoS ONE. 2016;11:e0150183. https://doi.org/10.1371/journ al.pone.0150183.

3. Mauchline TH, Malone JG. Life in earth-the root microbiome to the rescue? Curr Opin Microbiol. 2017;37:23-8. https://doi.org/10.1016/j. mib.2017.03.005.

4. Lozano GL, Bravo Jl, Handelsman J. Draft genome sequence of Pseudomonas koreensis CI12, a Bacillus cereus "Hitchhiker" from the soybean rhizosphere. Genome Announc. 2017;5:e00570. https://doi.org/10.1128/ genomeA.00570-17.

5. Lopes LD, Pereira E, Silva MC, Weisberg AJ, Davis EW 2nd, Yan Q, et al. Genome variations between rhizosphere and bulk soil ecotypes of a Pseudomonas koreensis population. Environ Microbiol. 2018;20:4401-14. https://doi.org/10.1111/1462-2920.14363.

6. Shahi N, Mallik SK. Recovery of Pseudomonas koreensis from eye lesions in golden mahseer, Tor putitora (Hamilton, 1822) in Uttarakhand. India. J Fish Dis. 2014;37:497-500. https://doi.org/10.1111/jfd.12126.

7. Kaur M, Jangra M, Singh H, Tambat R, Singh N, Jachak SM, Mishra S, Sharma C, Nandanwar H, Pinnaka AK. Pseudomonas koreensis recovered from raw yak milk synthesizes a $\beta$-carboline derivative with antimicrobial properties. Front Microbiol. 2019;10:1728. https://doi.org/10.3389/fmicb 2019.01728.

8. Kwon SW, Kim JS, Park IC, Yoon SH, Park DH, Lim CK, et al. Pseudomonas koreensis sp. nov., Pseudomonas umsongensis sp. nov. and Pseudomonas jinjuensis sp. nov., novel species from farm soils in Korea. Int J Syst Evol Microbiol. 2003:53:21-7. https://doi.org/10.1099/ijs.0.02326-0.

9. Hultberg M, Alsberg T, Khalil S, Alsanius B. Suppression of disease in tomato infected by Pythium ultimum with a biosurfactant produced by Pseudomonas koreensis. Biocontrol. 2010;55:435-44. https://doi. org/10.1007/s10526-009-9261-6.

10. Toribio J, Escalante AE, Caballero-Mellado J, González-González A, Zavala S, Souza V, et al. Characterization of a novel biosurfactant producing Pseudomonas koreensis lineage that is endemic to Cuatro Ciénegas Basin. Syst Appl Microbiol. 2011;34:531-5. https://doi.org/10.1016/j.syapm 2011.01 .007$.
11. Richter M, Rosselló-Móra R. Shifting the genomic gold standard for the prokaryotic species definition. Proc Natl Acad Sci USA. 2009;106:1912631. https://doi.org/10.1073/pnas.0906412106

12. Richter M, Rosselló-Móra R, Glöckner FO, Peplies J. JSpeciesWS: a web server for prokaryotic species circumscription based on pairwise genome comparison. Bioinformatics. 2016;32:929-31. https://doi.org/10.1093/ bioinformatics/btv681.

13. Garrido-Sanz D, Arrebola E, Martínez-Granero F, García-Méndez S, Muriel C, Blanco-Romero E, et al. Classification of isolates from the Pseudomonas fluorescens complex into phylogenomic groups based in group-specific markers. Front Microbiol. 2017;8:413. https://doi.org/10.3389/fmicb .2017.00413.

14. Beaton A, Lood C, Cunningham-Oakes E, MacFadyen A, Mullins AJ, Bestawy WE, et al. Community-led comparative genomic and phenotypic analysis of the aquaculture pathogen Pseudomonas baetica a390T sequenced by lon semiconductor and Nanopore technologies. FEMS Microbiol Lett. 2018;365:fny069. https://doi.org/10.1093/femsle/fny069.

15. Overbeek R, Olson R, Pusch GD, Olsen GJ, Davis JJ, Disz T, et al. The SEED and the rapid annotation of microbial genomes using subsystems technology (RAST). Nucl Acids Res. 2014;42(Database issue):D206-14. https:// doi.org/10.1093/nar/gkt1226.

16. Seemann T. Prokka: rapid prokaryotic genome annotation. Bioinformatics. 2014;30:2068-9. https://doi.org/10.1093/bioinformatics/btu153.

17. Zankari E, Hasman H, Cosentino S, Vestergaard M, Rasmussen S, Lund $\mathrm{O}$, et al. Identification of acquired antimicrobial resistance genes. J Antimicrob Chemother. 2012;2012(67):2640-4. https://doi.org/10.1093/jac/ dks261.

18. Lin H, Hu S, Liu R, Chen P, Ge C, Zhu B, et al. Genome sequence of Pseudomonas koreensis CRS05-R5, an antagonistic bacterium isolated from rice paddy field. Front Microbiol. 2016;7:1756. https://doi.org/10.3389/ fmicb.2016.01756.

19. Mittal R, Sharma S, Chhibber S, Aggarwal S, Gupta V, Harjai K. Correlation between serogroup, in vitro biofilm formation and elaboration of virulence factors by uropathogenic Pseudomonas aeruginosa. FEMS Immunol Med Microbiol. 2010;58:237-43. https://doi.org/10.1111/j.1574$695 \times 2009.00627 \times$.

20. Sun YY, Chi H, Sun L. Pseudomonas fluorescens filamentous hemagglutinin, an iron-regulated protein, is an important virulence factor that modulates bacterial pathogenicity. Front Microbiol. 2016;7:1320. https:// doi.org/10.3389/fmicb.2016.01320.

21. Murphy TF, Brauer AL. Expression of urease by Haemophilus influenzae during human respiratory tract infection and role in survival in an acid environment. BMC Microbiol. 2011;11:183. https://doi. org/10.1186/1471-2180-11-183.

22. Filloux A, Michel G, Bally M. GSP-dependent protein secretion in gramnegative bacteria: the Xcp system of Pseudomonas aeruginosa. FEMS Microbiol Rev. 1998;22:177-98. https://doi.org/10.1111/j.1574-6976.1998. tb00366.x.

23. Douzi B, Filloux A, Voulhoux R. On the path to uncover the bacterial type II secretion system. Philos Trans R Soc Lond B Biol Sci. 2012;367:1059-72. https://doi.org/10.1098/rstb.2011.0204.

24. Sana TG, Flaugnatti N, Lugo KA, Lam LH, Jacobson A, Baylot V, et al. Salmonella typhimurium utilizes a T6SS-mediated antibacterial weapon to establish in the host gut. Proc Natl Acad Sci USA. 2016;113:E5044-51. https://doi.org/10.1073/pnas.1608858113.

25. Dingemans J, Ghequire MG, Craggs M, De Mot R, Cornelis P. Identification and functional analysis of a bacteriocin, pyocin $\mathrm{S6}$, with ribonuclease activity from a Pseudomonas aeruginosa cystic fibrosis clinical isolate. Microbiologyopen. 2016;5:413-23. https://doi.org/10.1002/mbo3.339.

\section{Publisher's Note}

Springer Nature remains neutral with regard to jurisdictional claims in published maps and institutional affiliations. 